\title{
Sialic acid and glycosaminoglycans as biomarkers in dental fluorosis and periodontal diseases
}

\begin{abstract}
The disease severity of periodontal disease and its phases of activity and remission are not able to be detected by the routine clinical and radiographic methods. The oral diagnostic fluids like saliva, GCF, serum and plasma has served as a good source for identification of biomarkers in periodontitis. However every method has its own advantage and disadvantage. The various biomarkers in GCF have been a good source for examination and correlation of different phases of periodontal health and disease specifically in a non-invasive mode. There are several biochemical markers assessed in periodontitis patients (with or without fluorosis) to ascertain indirectly the degree of destruction of periodontal tissues. Glycosaminoglycans (GAG) and Sialic Acid (SA) are the two important biomarkers of GCF which are used for assessment of periodontal destruction and defence activity respectively. This paper reviews on the biologic functions of Sialic acid and Glycosaminoglycans and the studies related to these two biomarkers.
\end{abstract}

Volume 5 Issue 2 - 2020

\author{
Dr Aswin Prasad S, Dr Liya Anil, Dr K L \\ Vandana \\ Department of Periodontics, College of dental Sciences, Rajiv \\ Gandhi University, India
}

Correspondence: DrVandana KL, MDS, Senior Professor Department of Periodontics, College of dental Sciences, Rajiv Gandhi university, Davangere-577 004, Karnataka, India, Tel (08192) 231285, 231029, Email vanras@gmail.com

Received: February 18, 2020 | Published: June 17, 2020

Keywords: fluororsis, periodontitis, gingival crevicular fluid, serum, saliva, sialic acid, glycosaminoglycans

\section{Introduction}

The potential risk for periodontal disease can be identified and quantified by estimating biomarkers in the forefront by the diagnostic researches in oral and periodontal disease. ${ }^{1}$ There are several biochemical markers assessed in periodontitis patients (with or without dental fluorosis) to ascertain indirectly the degree of destruction of periodontal tissues The host derived oral diagnostic fluid such as saliva, Gingival crevicular fluid (GCF) as well as blood components like plasma and serum serve as a good source for detection of biomarkers in periodontal disease activity. There are own advantages and limitations for each of these diagnostic fluids. Sialic acid (SA), a biomarker with its biological activity as a defence molecule against oxidative stress would serve as an important marker in GCF to ascertain the host immune response. It also regulates innate immunity, limits injury and aids in healing as defence molecule against oxidative stress in inflammatory disease such as periodontitis. ${ }^{2}$ While the GAG( glycosaminoglycans), an important structure entity of periodontal bone provides a marker for bone destruction. In a given clinical situation the estimation of biomarkers more than one based on host immunity and tissue breakdown products complements the clinical diagnosis and prognosis.

Dental fluorosis subjects with periodontitis was found to be in higher occurrence and the Sialic acid along with GAG have been estimated in few of the experimentally induced and naturally induced florist rabbits and humans. Susheela et al have reported decreased sialic acid to GAG levels in rabbits with intentionally induced fluorosis. So far only one human study has been done by Vandana KL which had conveyed the enhancing role of biochemical parameters such as SA and chondroitin sulfate in fluorosis periodontal disease status and non fluorosed periodontitis patients. The objective of this paper is to review the biologic functions of Sialic acid and Glycosaminoglycans and the studies related to these two biomarkers.

\section{Biomarkers}

There are several biochemical markers assessed in periodontitis patients (with or without fluorosis) to ascertain indirectly the degree of destruction of periodontal tissues. Of these, Glycosaminoglycans (GAG) have been identified as one of the many biochemical components of GCF. ${ }^{3}$ Patients with periodontitis may have elevated circulating levels of specific inflammatory markers that can be correlated to the severity of the disease. Significantly there are higher levels of salivary free sialic acid in chronic periodontitis compared to healthy controls ${ }^{4}$ GAG and SA are the two important biomarkers of GCF which are used in assessment of periodontal destruction and defence activity.

\section{Gag as a gcf biomarker}

GAGs are heterogeneously negatively charged polysaccharide structures of bone matrix consists of uronic acid with hexosamine and are combined with different proteins in various connective tissues of organs. The various GAG types in GCF are chondroitin sulfate, heparansulfate, dermatansulfate, hyaluronic acid found throughout the connective tissues of alveolar bone, cementum, gingiva and periodontal ligament. The sulphated GAG is found to be less in healthy periodontium. ${ }^{5}$

In gingival epithelium, $60 \%$ of GAG is heparin sulphates, $60 \%$ dermatan sulphate and chondratan sulphate is present as major component in gingival connective tissue. The major component of GAG in alveolar bone is $90 \%$ chondratan sulphate. ${ }^{2,6-8}$ The literature review on salivary, serum and GCF GAG and fluorosis and GAG are depicted in Tables $1 \& 2$ respectively. 
Table I Literature review on salivary, serum and GCF GAG in periodontitis

\begin{tabular}{|c|c|c|}
\hline SI no & Author & Methodology \\
\hline \multicolumn{3}{|c|}{ Salivary GAG: no studies reported so far } \\
\hline \multicolumn{3}{|c|}{ Serum GAG : } \\
\hline I & Jha $M$ et al., ${ }^{24}$ & $\begin{array}{l}\text { In an animal study, after excessive ingestion of } \\
\text { fluoride, the significance of } S A \text { : GAG in the serum of } \\
\text { rabbit and human subjects were assessed }\end{array}$ \\
\hline 2 & Susheela AK et al., ${ }^{25}$ & $\begin{array}{l}\text { The circulating levels of SA ( } \mathrm{N} \text {-acetylneuraminic } \\
\text { acid) and GAG were measured by winzler's method } \\
\text { and alcian blue method in } 69 \text { patients with spinal } \\
\text { disorders of orthopaedic interest (ankylosing } \\
\text { spondylitis I7, osteofluorosis } 6 \text {, idiopathic backache } \\
\text { I0, osteoarthrosis } 16 \text {, osteoporosis } 20 \text { ) to analyse } \\
\text { the expected changes in the bone contents }\end{array}$ \\
\hline
\end{tabular}

Inference

GCF GAG:

I Last KS et al., ${ }^{10}$

$2 \quad$ Smith AJ et al., ${ }^{12}$

Okazaki J et al., 5

Khongkhunthian S et al.,22 which is recognized by WF6 monoclonal antibody, in GCF from different stages of periodontal disease and healthy periodontium

measured the levels of hCAPI8/LL-37 in GCF from patients with periodontal diseases compared

Makeudom A et al., ${ }^{18}$ measured the GAG levels taking Chondroitin 4 sulfate as the standard in Human GCF from subjects of Chronic gingivitis, Early chronic periodontitis, Advanced chronic periodontitis, Treated sites of periodontal disease and Periodontal surgery using electrophoresis

investigated levels of hyaluronan and chondroitin-4 sulphate in the GCF of patients with chronic adult periodontitis at diseased and healthy sites before and after treatment using glass micropipettes and analysed for GAG content by cellulose acetate electrophoresis

conducted a study on the levels of sulfatedglycosaminoglycans in GCF based on dimethylmethylene blue dye assay determined the correlation between hCAPI8/LL-37 and CS levels in patients with periodontitis

Enhanced levels of GAG occured in rabbit and human sera after fluoride intoxication. More than $50 \%$ reduction in the ratio of SA : GAG was observed in rabbit sera. ${ }^{24}$

suggested that the SA/GAG ratio can be used as a diagnostic test in ankylosing spondylitis as there was a $37 \%$ decrease in the mean ratio of SA/GAG noted in osteofluorosis when compared with control values which was half the value of Ankylosi5ng spondylitis. ${ }^{25}$

observed that the non-sulphated GAG, hyaluronic acid, was present in all samples and was the only major band from sites of chronic gingivitis. ${ }^{10}$

confirmed the use of the sulphated glycosaminoglycan chondroitin-4-sulphate as a potential diagnostic aid of periodontal tissue destruction. ${ }^{12}$

concluded that, the major constituent of GAG whose monomers comprised of a core protein to which oligisaccharides and large number of GAG chains are such as CS along with non sulfatedhyaluron present in GCF of severely inflamed periodontal sites. However sulfatedglycosaminoglycans was found to be less in healthy periodontium. ${ }^{5}$

concluded that Elevated CSWF6 epitope levels in GCF are associated with severity of periodontitis and the WF6 antibody may therefore be clinically applied to monitor disease severity and progression. ${ }^{22}$

found significant correlations between the hCAPI8/ LL-37 and the CS levels in CP but not in aggressive periodontitis. ${ }^{18}$

Table 2 Literature review on fluorosis and GAG

\begin{tabular}{|c|c|c|c|}
\hline SI no & Author & Methodology & Inference \\
\hline I & $\begin{array}{l}\text { Mohan Jha et } \\
\text { al., }{ }^{26}\end{array}$ & $\begin{array}{l}\text { In an animal study, the urinary excretion of GAG, } \\
\text { hydroxyproline and hydroxylysine in rabbits after excessive } \\
\text { ingestion of fluoride were assessed }\end{array}$ & $\begin{array}{l}\text { observed that the diminished urinary excretion of } \\
\text { GAG in human and rabbit enhances the serum GAG } \\
\text { levels. }{ }^{26}\end{array}$ \\
\hline 2 & $\begin{array}{l}\text { Sharma K et } \\
\text { al., }{ }^{27}\end{array}$ & $\begin{array}{l}\text { In an animal study, effect of fluoride on molecular weight, } \\
\text { charge density and age related changes in the sulphated } \\
\text { isomers of GAG of the rabbit cancellous bone were assessed }\end{array}$ & $\begin{array}{l}\text { concluded that the decreased circulatory levels of SA } \\
\text { was noted in experimentally induced fluorosis but } \\
\text { GAG levels were found to be increased. }{ }^{27}\end{array}$ \\
\hline
\end{tabular}




\section{GAG and early onset periodontitis}

In early onset periodontitis, the various bacterial virulence factors (enzymes and lipopolysaccharide) degrade the PGs and GAG to a greater extent. ${ }^{9}$ and host generated mechanisms special free oxygen radicals along with enzymes contribute to the GAG depolymerisation. Further studies are required to comprehend the extra cellular matrix breakdown in early onset periodontitis.

\section{Various condition where in GCF GAG is detected}

The mineralization changes in bone, bone remodelling, trauma from occlusion, orthodontic forces and extracted tooth healing events in the periodontium alters the GAG level in GCF. ${ }^{10,11}$ GAG in GCF is seen during wound healing after implant insertion ${ }^{4,12}$ In all these situations, the presence of $\mathrm{C} 4 \mathrm{~s}$ is said to be due to underlying tissue remodelling.

\section{GAG changes after surgery and healing events}

The presence of GAG following surgery is said to be with a molecular identity intermediate between CS and DS which were noted to increase past one week and decreased in the following 4 weeks. The similar observations was found for sulphated GAG. ${ }^{10}$ During postoperative healing, the expression of CS in healing wound appears to decrease followed by gradual increase coinciding with the appearance and maturation of granulation tissue. ${ }^{13}$ The CS levels begins to increase as this tissue matures and remodel. This situation of increased CS levels during remodelling as a part of normal physiological event or during healing needs to be discriminated from increased CS levels during tissue destruction.

Irrespective of CS source, it is addressed that return of GCF CS to baseline levels during healing period is suggestive of the matured state of the wounds based on the "rebound" phenomenon of CS that is initial increase during healing to decreased levels as tissue is matured. However, if this rebound of CS don't occur, that is the CS level continue to increase without coming back to baseline, then it would be suggestive of continued infection or unsatisfactory resume to treatment. This role of CS has clinical significance while measured at different time periods than merely its actual levels.

\section{SA as a gcf biomarker}

A nine-carbon sugar acid called as Sialic acid (SA), is present predominantly at terminal positions of surface-exposed glycoconjugates of eukaryotic cells and these acids confer important properties on those cell surfaces. ${ }^{14,15}$ The glycoproteins are the important component of salivary proteins, the carbohydrate disaccharide chains (SA) of these glycoproteins provides resistance to proteolytic degradation. ${ }^{16} \mathrm{SA}$ has a role in various enzyme action and in binding of toxins. ${ }^{17}$ The important functions of SA include regulation of innate immunity, limiting tissue injury, aids in healing as defence molecule against oxidative stress in inflammatory diseases including periodontitis. ${ }^{2}$

The role of SA as a diagnostic marker of periodontal destruction by the reactive oxygen radicals by oxidative stress is gaining popularity and can be addressed as an antioxidant marker. The cleavage of glycosidic linkage as a result of hydrolysis of terminal SA of mucin releases SA into tissue environment. The sialidase enzyme increases free SA level in saliva. ${ }^{6}$ A number of periodontal pathogens produce sialidases and that produced by $\mathrm{T}$ forsythia is said to promote biofilm formation as well it serves as an indicator for TF. In periodontitis the sialidase activity is higher than gingivitis and its levels are correlated with clinical parameters.

The elevated levels of TSA in saliva and serum is reported which is suggestive of protective role of SA in periodontitis. The increased TSA levels might be regarded as a defensive molecule against the increased oxidative stress in inflammatory disease like periodontitis. ${ }^{2}$

\section{Fluorosis and SA}

Fluoride toxication results from increasing fluoride ions, which lead to inhibition of glycoprotein synthesis. ${ }^{8}$ Some of the glycoproteins in serum are $\alpha-1$ acid protein (orosomukoid), aantitrypsin, haptoglobulin, ceruloplasmine, fibrinogen, and transferring. ${ }^{4,10}$ Carbohydrates found in the structure of serum glycoproteins are hexose, hexosamine, fucose, and sialic acid. ${ }^{11}$ Some glycol proteins are involved in normal biological calcification of bone and teeth. Because of the calcium binding property of SA, for determining the glycoprotein levels in health and disease, SA levels have been used as a marker. It plays a role in cell-cell recognition, protein targeting, protease resistance, conformational stabilization, adhesion, and intracellular signalling events in biological systems. ${ }^{18}$ Increase of SAconcentration have been reported in cardiovascular diseases, cancer, diabetes, patients with chronic glomerulonephritis, and chronic renal failure. The level of blood serum SA in health and illness situations is evaluated as a marker for glycoprotein amount. Table 3 represents the literature review on fluorosis and SA.

Table 3 Literature review on fluorosis and SA

\begin{tabular}{|c|c|c|c|}
\hline SI no & Author & Method & Inference \\
\hline I & $\begin{array}{l}\text { Susheela AK et } \\
\text { al., }^{24}\end{array}$ & $\begin{array}{l}\text { assessed the levels of SA and GAG in the sera of } \\
\text { rabbit and human subjects who ingested fluoride } \\
\text { and had clinical manifestation of fluorosis }\end{array}$ & $\begin{array}{l}\text { reported decreased circulating SA to GAG levels in rabbits } \\
\text { with intentionally induced fluorosis concluding that level of } \\
\text { these chemical constituents in sera possibly reflect changes } \\
\text { occurring in calcified and noncalcified tissues due to fluoride } \\
\text { intoxication. }{ }^{24}\end{array}$ \\
\hline 2 & $\begin{array}{l}\text { Martins-Gomes } \\
\text { AM et al., }{ }^{28}\end{array}$ & $\begin{array}{l}\text { In a study, parameters such as the flow rate, } \\
\text { buffer capacity, SA, protein and electrolyte } \\
\text { concentrations, and amylase and peroxidase } \\
\text { activities were analyzed in stimulated whole } \\
\text { saliva from adolescents with dental fluorosis, } \\
\text { from I35 adolescents. }\end{array}$ & $\begin{array}{l}\text { The peroxidase activity and SA concentration showed some } \\
\text { differences compared to the non fluorosed controls. }{ }^{28}\end{array}$ \\
\hline
\end{tabular}




\section{Role of GCF to access the diagnostic markers}

Analysis of the constituents of gingival crevicular fluid (GCF) provides a non-invasive method of obtaining information regarding the site-specific state of the underlying periodontium. Many different components within GCF have been examined in an attempt to correlate findings with differing states of periodontal health and disease. ${ }^{20,21}$ There are various ways to collect GCF like capillary method or micro pipettes, ${ }^{10,12,22}$ paper strips, ${ }^{23}$ paper points. ${ }^{24}$

\section{Disadvantages of gcf pooling}

Without pooling, the GCF samples can be analysed on a sitespecific basis and correlated with the clinical features of the same sites (pre and post treatment). The enzymes largely generated locally and based on the active enzyme levels found at the healthy sites, a systemic contribution would be minimal. However, the problems associated with site specific GCF sampling is that the small volume of GCF collected due to slow flow rates can clearly limit the detailed analysis of components . This problem is overcomed by GCF pooling. GCF collection time is varied in literature among different authors with $30 \mathrm{sec},{ }^{18} 5 \mathrm{~min},{ }^{12,22} 15-20 \mathrm{~min} .{ }^{5,10}$

An intense research efforts in diagnostic tests are stimulated as there is lack of predictive value in periodontal disease detection. At present, the clinical parameters for periodontal disease such as pocket depth, bleeding on probing and radiographic bone loss are representative of past disease activity. In the periodontal diagnosis, the biochemical analysis of GCF has gained its importance. ${ }^{22}$ The various studies related to salivary, serum and GCF SA in periodontitis have been described in Table 4. ${ }^{25-31}$

Table 4 Literature review on salivary, serum and GCF SA in periodontitis

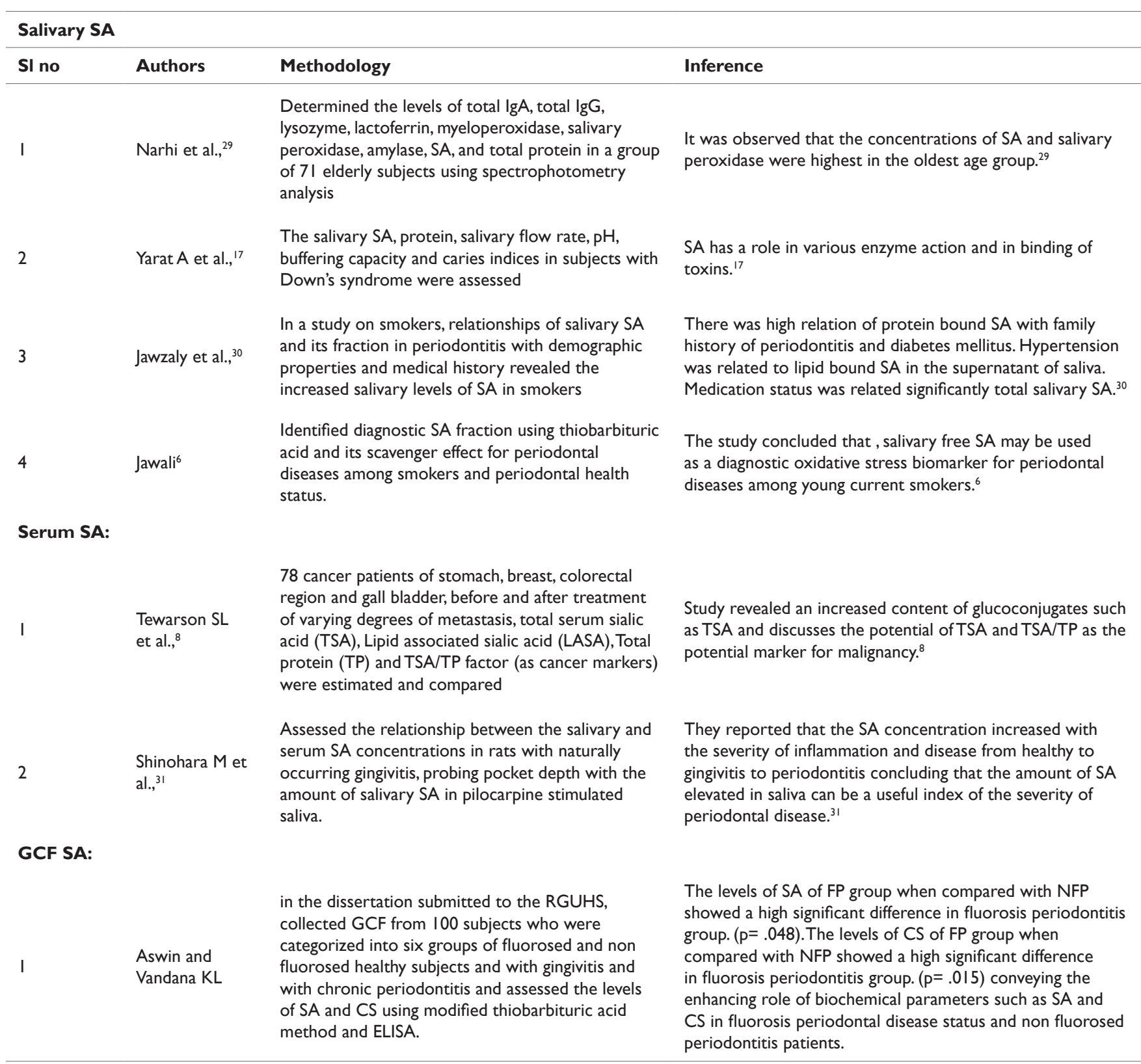




\section{Discussion}

There are several biochemical markers assessed in periodontitis patients (with or without fluorosis) to ascertain indirectly the degree of destruction of periodontal tissues. Of these, glycosaminoglycans (GAG) have been identified as one of the many biochemical components of GCF. ${ }^{3}$

Patients with periodontitis may have elevated circulating levels of specific inflammatory markers that can be correlated to the severity of the disease. Significantly higher levels of salivary free sialic acid in chronic periodontitis compared to healthy controls. ${ }^{4}$ The GAG and SA are two important biomarkers of GCF which are used or assessment of periodontal destruction and defence activity respectively. The role of SA as a diagnostic marker of periodontal destruction by the reactive oxygen radicals by oxidative stress is gaining popularity and essay can be addressed as an antioxidant marker. The elevated levels of TSA in saliva and serum is reported which is suggestive of protective role of SA in periodontitis. The increased TSA levels might be regarded as a defensive molecule against the increased oxidative stress in inflammatory disease like periodontitis. ${ }^{2}$

The glycosaminoglycans are heterogeneously negatively charged polysaccharide structures of bone matrix consists of uronic acid with hexosamine and are combined with different proteins in various connective tissues of organs. The GAG content as a part of matrix destruction derives its importance due to its biological actions. The various causes of breakdown or depolymerization of proteoglycan include catabolic and metabolic activity during inflammation, enzymes released by the inflammatory cells and fibroblast at inflamed and reactive oxygen species released by the polymorphonuclear neutrophils are able to begin matrix destruction during inflammation.

\section{Conclusion}

Considering the need, the analysis of biochemical markers in the GCF is in the forefront in diagnosis and prognosis of periodontal disease and its treatment.The oral diagnostic fluids like saliva, GCF, serum and plasma has served as a good source for identification of biomarkers in periodontitis. However every method has its own advantage and disadvantage. The various biomarkers in GCF have been a good source for examination and correlation of different phases of periodontal health and disease specifically in a non-invasive mode.

\section{Acknowledgments}

None.

\section{Conflicts of interest}

No conflicts of interest.

\section{References}

1. Dr. Varun Dahiya, Dr. Pradeep Shukla, Dr. Artika Sharma, et al. Host Derived Biomarkers in Periodontitis: an Insight. RRJDS. 2016;4

2. Rathod SR, Khan F, Kolte AP, et al. Estimation of salivary and serum total sialic Acid levels in periodontal health and disease. J Clin Diagn Res. 2014;8(9):19-21.

3. Embery G, Waddington R. Gingival crevicular fluid: Biomarkers of periodontal tissue activity. Adv Dent Res. 1994;8(2):329-336.

4. Inasu S, Thomas B, Kumari S, et al. Evaluation of serum and salivary sialic acid and nitric oxide levels in chronic periodontitis patients. Int JAppl Dent Sci. 2016;2(2):74-76.
5. Okazaki J, Komasa Y, Kamada A, et al. Measuring Sulfated Glycosaminoglycans in Gingival Crevicular Fluid Based on Dimethylmethylene Blue. Journal of Oral Tissue Engineering. 2006;4(1):51-56.

6. Jawzali JI. Association between salivary sialic acid and periodontal health status among smokers. The Saudi dental journal. 2016;28(3):124-135.

7. Lindberg G, Rastam L, Gullberg B, et al. Serum concentrations of total sialic acid and sialoglycoproteins in relation to coronary heart disease risk markers. Atherosclerosis. 1993;103(2):123-129.

8. Tewarson SL, Mittal VP, Singh M, et al. Serum sialic acid--an important cancer marker. Indian J Cancer. 1993;30(3):125-131.

9. Teles RP, Gursky LC, Faveri M, et al. Relationships between subgingivalmicrobiota and GCF biomarkers in generalized aggressive periodontitis. J Clin Periodontol. 2010;37(4):313-323.

10. Last KS, Stanbury JB, Embery G. Glycosaminoglycans in human gingival crevicular fluid as indicators of active periodontal disease. Arch Oral Biol. 1985;30(3):275-281.

11. Waddington RJ, Embery G, Last KS. Glycosaminoglycans of human alveolar bone. Arch Oral Biol. 1989;34(7):587-589.

12. Smith QT, Harriman L, Au GS, et al. Neutrophil elastase in crevicular fluid: comparison of a middle-aged general population with healthy and periodontitis groups. Journal of clinical periodontology. 1995;22(12):935941.

13. Bentley JP. Rate of chondroitin sulfate formation in wound healing. Ann Surg. 1965;165(2):186-191.

14. Severi E, Hood DW, Thomas GH. Sialic acid utilization by bacterial pathogens. Microbiology. 2007;153(Pt 9):2817-2822.

15. Roy S, Honma K, Douglas CI, et al. Role of sialidase in glycoprotein utilization by Tannerella forsythia. Microbiology. 2011;157(11):31953202 .

16. Gul SS, Douglas CWI, Griffiths GS. A pilot study of active enzyme levels in gingival crevicular fluid of patients with chronic periodontal disease. $J$ Clin Periodontol. 2016;43(8):629-636.

17. Yarat A, Akyuz S, Koc L, et al. Salivary sialic acid, protein, salivary flow rate, $\mathrm{pH}$, buffering capacity and caries indices in subjects with Down's syndrome. Journal of Dentistry. 1999;27(2):115-118.

18. Makeudom A, Kulpawaropas S, Montreekachon P, et al. Positive correlations between h CAP 18/LL-37 and chondroitin sulphate levels in chronic periodontitis. Journal of clinical periodontology. 2014;41(3):252261.

19. Curtis MA, Gillett IR, Griffiths GS, et al. Detection of high-risk groups and individuals for periodontal diseases: laboratory markers from analysis of gingival crevicular fluid. J Clin Periodontol. 1989;16(1):1-11.

20. Lamster IB. The host response in gingival crevicular fluid: potential application in periodontitis clinical trials. $J$ Periodontol. 1992;63(12S):1117-1123

21. Giannobile WV, Riviere GR, Gorski JP, et al. Glycosaminoglycans and periodontal disease: analysis of GCF by safranin O. J Periodontol. 1993;64(3):186-190.

22. Khongkhunthian S, Kongtawelert P, Ongchai S, et al. Comparisons between two biochemical markers in evaluating periodontal disease severity: a cross-sectional study. BMC Oral Health. 2014;14:107.

23. Guentsch A, Kramesberger M, Sroka A, et al. Comparison of gingival crevicular fluid sampling methods in patients with severe chronic periodontitis. Journal of periodontology. 2011;82(7):1051-1060.

24. Jha M, Susheela AK. In vivo chondrogenesis and histochemical appearance of dermatan sulphate in rabbit cancellous bone. Differentiation. 1982;22(3):235-236. 
25. Susheela AK, Das TK, Khurana JS, et al. Circulating levels of sialic acid and glycosaminoglycans: a diagnostic test for ankylosing spondylitis. Annals of the rheumatic diseases. 1988;47(10):833-837.

26. Mohan Jha, Jasbeer Koacher, AK Susheela. Urinary excretion of glycosaminoglycans, hydroxyproline and hydroxylysine in rabbits after excessive ingestion of fluoride. Clinical and Experimental Pharmacology \& Physiology. 1983;10(5):615-619.

27. Sharma K, Susheela AK. Effect of fluoride on molecular weight, charge density and age related changes in the sulphated isomers of glycosaminoglycans of the rabbit cancellous bone. International journal of tissue reactions. 1988;10(5):327-334.

28. Martins Gomes AM, Nicolau J, de Souza DN, et al. A study of some parameters in stimulated saliva from adolescents with dental fluorosis. Journal of oral science. 2001;43(4):225-231.
29. Narhi TO, Tenovuo J, Ainamo A, et al. Antimicrobial factors, sialic acid, and protein concentration in whole saliva of the elderly. European Journal of Oral Sciences. 1994;102(2):120-125.

30. Jawzaly JW, Hasan HG, Ahmed BM. Relationships of salivary sialic acid and its fraction in periodontitis with demographic properties and medical history. International Journal of Dental Science and Research Development. 2013;3(2):17-26.

31. Shinohara M, Ohura K, Ogata K, et al. The relation between the sialic acid concentrations in the serum and whole saliva in rats with naturally occuring gingivitis. Jpn J Pharmacol. 1994;64(1):61-63. 